\title{
Off-the-shelf immunotherapy could lead to more accessible cancer treatment
}

Immunotherapy is a type
of cancer treatment that
harnesses the immune system
to destroy cancerous cells.
Dr Li Zhang and colleagues
at Toronto General Hospital
Research Institute and the
University of Toronto, are
pioneering a new type of
"off-the-shelf" treatment
using a type of immune cell-a
double negative T (DNT)
cell. Dr Zhang's work shows
that DNT cells are a safe and
effective therapy to tackle
many different types of cancer.
Importantly, these cells can
be produced in large numbers
from healthy donors without
gene-editing and successfully
stored for future use.
is known as adoptive T cell therapy
(ACT). Recently, one kind of ACT called Chimeric Antigen Receptor T (CART) cells has been proven to be a very effective treatment for patients with more, and better, treatments available than ever before. Chemotherapy and radiation are perhaps the most some forms of leukaemia. However, as cancer, but another type of treatmen is also showing great promise: immunotherapy. Immunotherapy is already an established treatment route for several types of cancer, including certain types of haematologic and solid tumours and is also being trialled in many other forms of the disease.

Immunotherapy works by utilising the immune system to recognise and attack cancerous cells. There are different forms of immunotherapy, each of which uses a different aspect

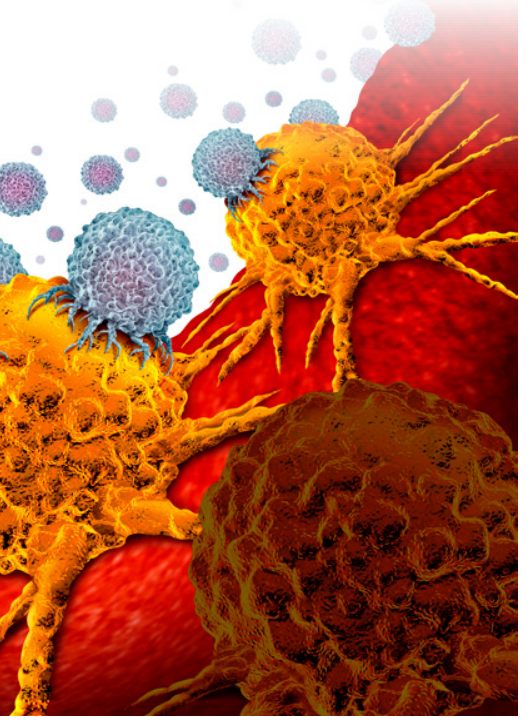
Administration approved CART treatment with patients receiving $T$ cells for them. This helps to avoid graftversus-host disease, a serious condition the tissues of the recipient's body.

Unfortunately, this patient-specific form of ACT is expensive and manufacturing failures, making the treatment inaccessible for some patients. Further, as the $T$ cells are the needs of particular patients, there can be a delay in starting treatment during which the disease may progress.

"OFF-THE-SHELF"

MMUNOTHERAPY

The problems with ACT in its curren form could be solved by "off-the shelf "immunotherapy. This involves producing large quantities of $\mathrm{T}$ cells stored until they are needed, meaning
that patients should not have to wait
Double-negative T cells (DNT) as an off-the-shelf cancer therapy

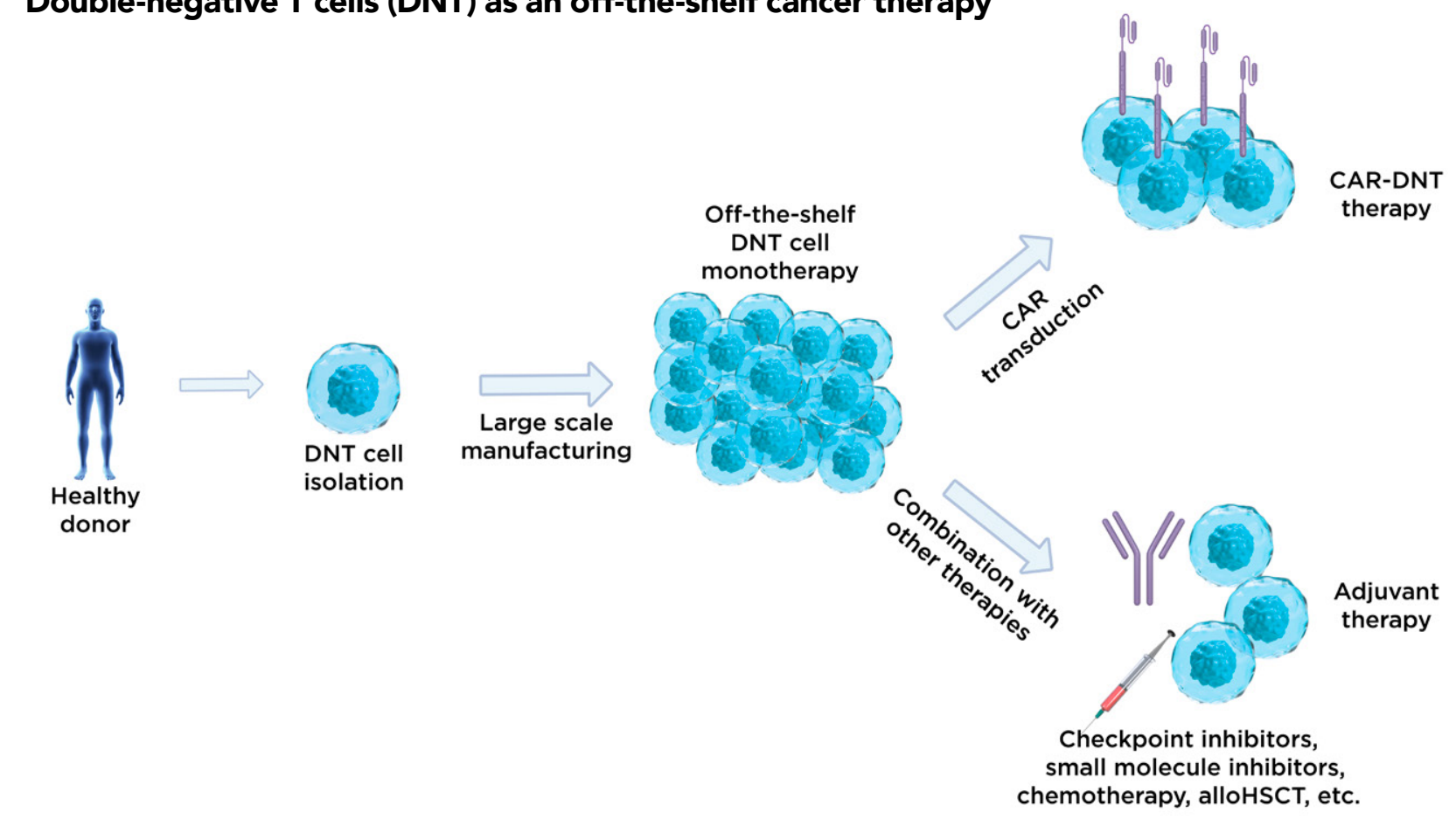
created, at a lower cost. A future offcolleagues, the researchers have show timitations in the current form of the Currently, the U.S. Food and Drug nechnically complex. The methods are complicated and can result in produced on demand in response to Priginating from heal hy donors and for treatment. This mass production approach would also allow a more the-shelf treatment must be accessible, providing a "one size fits all" treatment that has the potential to benefit many cancer sufferers. However, before this can happen, there are many challenges that must be overcome.

Dr Li Zhang is a senior scientist at the Toronto General Hospital Resear Institute and a professor at the University of Toronto. Together with her

initial research into double-negative $T$ must also be able to tolerate storage until they are needed. called double-

negative becaus

they lack certain

cellular markers

DNTs originating from just a single

present on

donor cou

RESEARCH PROVES THE PROMIS OF DNTS AS AN OFF-THE-SHELF CANCER THERAPY

Together with collaborators, Dr Zhang's lab have developed a method to produce large numbers of clinical-grade DNTs. The DNT cells were taken from healthy donors and then expanded (ie. clinically-compliant conditions. clinically-compliant conditions.

The anti-cancer abilities of DNTs were number of different

patients, with many different types of cancer: a key requirement for a true offthe-shelf treatment.

Dr Zhang and her team also discovered Dat cryopreservation, in which the DNTs were frozen more than 600 days before being thawed, did not affect the anti-cancer function of the cells. on-demand basis.

A major benefit of personalised ACT is that as it uses the patient's own cells, disease (where the recipient's immune system attacks the donor cells). Previous and others has show destroy cancer cells without triggering animal tests these cells effectively treat leukaemia, lung cancer and lymphoma. graft-versus-host disease. This is different cancers including lung cancer, The results were clear: the DNTs were supported by the results of Dr Zhang's lymphoma, and leukaemia, without proven to be toxic against all types of recent study, demonstrating that DNTs damaging normal cells.

However, before the next step towards an off-the-shelf treatment can be taken, further study was needed to demonstrate that DNTs are both safe harar numbers. Further, the cells cancer cell tested. Significantly, DNTs originating from just a single donor could successfully tackle many different types of cancer. At the same time, the normal tissue. cells. This discovery raises hopes that a single heatthy donor could supply produced from healthy donors will

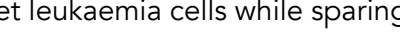

For DNTs to be a viable off-the-shelf treatment option, they also need to condition of host-versus-graft rejection. . prould allow the treatment to be that DNTs are able to 


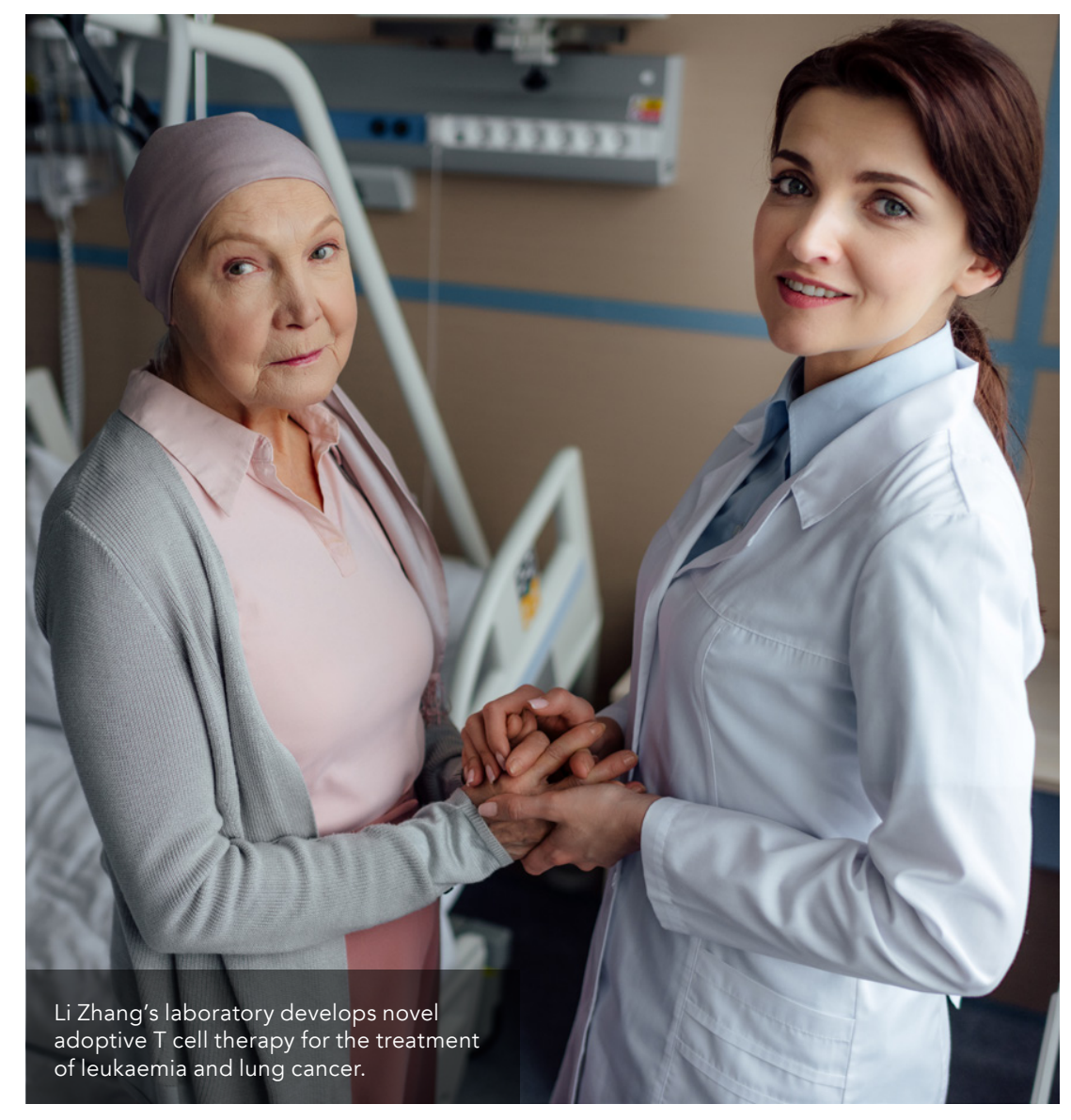

A future off-the-shelf treatment must be accessible, providing a "one size fits all" treatment that has the potential to benefit many cancer sufferers.

If this occurred, the DNTs would be swiftly wiped out by the recipient's efficacy short-lived Interestingly, Dr Zhang's findings suggest that DNTs somehow able to evade the recipient's immune response. How DNTs manage to do this remains unclear. However, this does mean that DNTs seem unlikel to trigger a host-versus-graft reaction.

Finally, Dr Zhang found that DNTs persist well within the recipient's body. Research on another group of immune cells, known as natural killer (NK) cells, found that, although the NK cells successfully attacked cancerous cells and did no harm to healthy tissue, they disappea from the patients body within a few weeks. This suggests that the patient's innor syst However, in Dr Zhed

research, DNTs survived in the presence of allogeneic immune cells for at least our weeks in an animal model which human cells so suer environment for to evade the recipient's immune systen and persist longer in patients, they could be a more effective treatment than NKs.

VERSATILITY OF DNT THERAPY Combining a new therapy with existing therapeutic approaches increases the chance of improving the patient outcome while minimising the risk of losing the benefits of established the that arise from cocts and new toxicities that arise from combination therapy. Using DNTs as an off-the-shelf therapy significantly increases the treatment flexibility therefore combining DNT herapies becomes possible. Dr Zhang that DNTs can synergise with other established cancer treatments such conventional chemotherapy, checkpoint and small molecule inhibitors to yield in a superior response in animal tests without increased toxicities. Further, Dr Zhang's group recently demonstrated the feasibility of using DNTs as a platform for a CAR technology. CAR technology has been the key for the unprecedented success of $\mathrm{ACT}$ in treating some leukaemia, but its wide clinical application is challenged by limitations associated with the

highly personalised manufacturing process. Combining CAR with DNTs pelf CAR-ACT product that is safe, shelf CAR-ACT product that is safe, Collectively, off-the-shelf DNT therepy Collectively, of the and accessible aption that can be incorporated with other treatment regimens to yield in a superior outcome in cancer.

A NEW, MORE ACCESSIBLE CANCER TREATMENT

Any new medical treatment must be proven to be both safe and effective. . of translation into widespread use; treatment which is too expensive, too complex, or too time-consuming is unlikely to benefit many patients. These factors currently limit the

For the first time, Dr Zhang and her eam have identified a T cell therapy that has the potential to be developed into a successful off-the-shelf treatmen for cancer. This research has shown at DNTs meet all the requirements of accessible ACT: they are easy to produce on a large scale and so are relatively less expensive; they can be preserved in advance with no effect different types of cancer; and they cause no harm to healthy tissues. With these advantages, DNTs offer the hop of an accessible, effective treatment for many cancer patients. Currently, the safety and efficacy of allogeneic DNT therapy in human recipients are being investigated in two phase ChicTR1900022795).

\section{A Behind the Research}

Dr Li Zhang

E: li.zhang@uhnresearch.ca T: +1 416-581-7521

\section{Research Objectives}

Research in Li Zhang's laboratory is focused on two main areas: developing novel adoptive $T$ cell therapy for the the mechanisms involved in immunity and tolerance and their relevance in disease.

\section{Detail}

\section{Li Zhang}

Princes Margaret Cancer Research Tower,

Toronto, Ontario, M5G, 1L7, Canad

Bio

Dr Zhang is a Professor in the Departments of Laboratory Medicine and Pathobiology and Immunology at the University of Toronto and senior scientist at University Health Network. Her research has been focused on understanding the cellular and molecular mechanisms involved in immunity and tolerance and developing new treatments for cancer, graft versus host disease and allograft rejection.

Funding

of Health Research, Canadion Cancer Society, Leukemia Lymphoma Society

\section{Collaborators}

- Dr Jong Bok Lee

- Dr Liming Yang

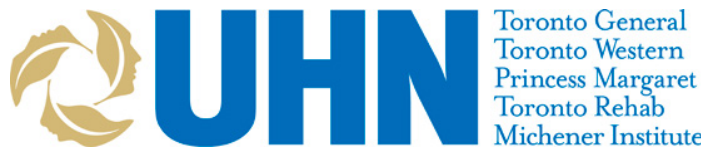

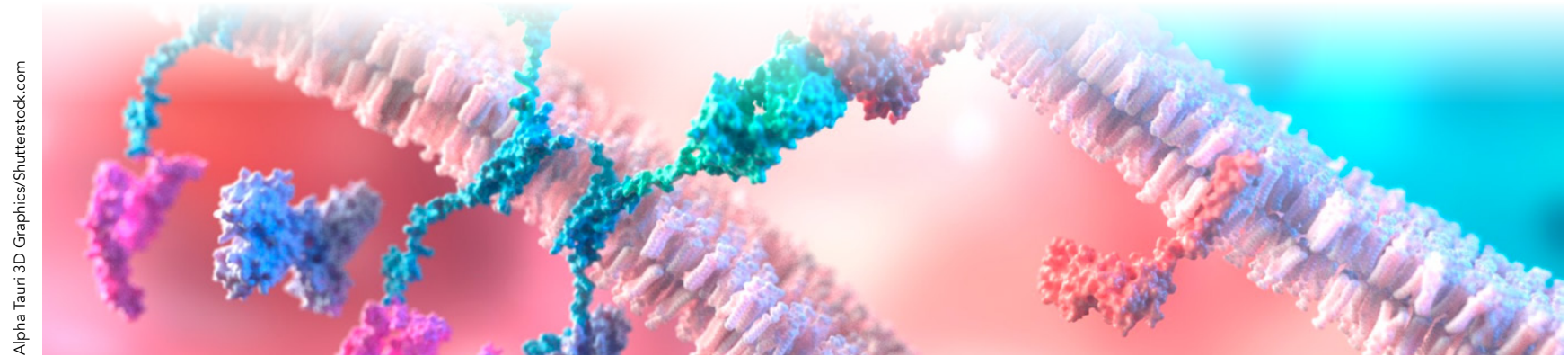

\section{References}

Lee, JB, et al. (2019) Developing allogeneic doublenegative $T$ cells as a novel off-the-shelf adoptive cellular therapy for cancer. Clincal Cancer Research 25(7):2241-

Fang, L. et al. (2019) Targeting late-stage non-small cell lung cancer with a combination of DNT cellular therapy and PD-1 checkpoint blockade. J Exp Clin Can

Chen, B. et al. (2018) Targeting chemotherapy-resistan leukemia by combining DNT cellular therapy with conventional chemotherapy. J Exp Clin Can Res. 37:88. https://doi.org/10.1186/s13046-018-0756-9

Vasic, D. et al. (2019) Developing a platform for "offthe-shelf" CAR-T cell therapies for cancer using double negative T cells. 2019 TFRI Symposium: Novel Cancer Target and Emerging Therapies.

\section{Personal Response}

What now needs to happen before DNTs can become an approved treatment for cancer patients?

II The safety and efficacy of DNT therapy is currently being investigated in two phase I clinical trials. (ChiCTR1900022795). Given the limitations of preclinical models, we need to determine the safety, efficacy, and persistence of infused allogeneic DNTs from these trials. Also, further investigations are needed on the mechanism of cancer-specific killing activity of DNTs to understand why some cancer cells are more susceptible 2253. doi:10.1158/1078-0432.CCR-18-229 